\title{
Asbestos-Induced Cellular and Molecular Alteration of Immunocompetent Cells and Their Relationship with Chronic Inflammation and Carcinogenesis
}

\author{
Hidenori Matsuzaki, ${ }^{1}$ Megumi Maeda, ${ }^{1,2}$ Suni Lee, ${ }^{1}$ Yasumitsu Nishimura, ${ }^{1}$ \\ Naoko Kumagai-Takei, ${ }^{1}$ Hiroaki Hayashi, ${ }^{1,3}$ Shoko Yamamoto, ${ }^{1}$ Tamayo Hatayama, ${ }^{1}$ \\ Yoko Kojima, ${ }^{4}$ Rika Tabata, ${ }^{4}$ Takumi Kishimoto, ${ }^{4}$ Junichi Hiratsuka, ${ }^{5}$ and Takemi Otsuki ${ }^{1}$ \\ ${ }^{1}$ Department of Hygiene, Kawasaki Medical School, 577 Matsushima, Kurashiki 7010192, Japan \\ ${ }^{2}$ Department of Biofunctional Chemistry, Division of Bioscience, Okayama University Graduate School of \\ Natural Science and Technology, 3-1-1 Tsushima-Naka, Okayama 7008530, Japan \\ ${ }^{3}$ Department of Dermatology, Kawasaki Medical School, 577 Matsushima, Kurashiki 7010192, Japan \\ ${ }^{4}$ Research Center for Asbestos-Related Diseases, Okayama Rosai Hospital, 1-10-25 Chikko-Midorimachi, Minami-Ku, \\ Okayama 7028055, Japan \\ ${ }^{5}$ Department of Radiation Oncology, Kawasaki Medical School, 577 Matsushima, Kurashiki 7010192, Japan
}

Correspondence should be addressed to Takemi Otsuki, takemi@med.kawasaki-m.ac.jp

Received 30 June 2011; Accepted 3 November 2011

Academic Editor: Vassilis Gorgoulis

Copyright ( 2012 Hidenori Matsuzaki et al. This is an open access article distributed under the Creative Commons Attribution License, which permits unrestricted use, distribution, and reproduction in any medium, provided the original work is properly cited.

\begin{abstract}
Asbestos causes lung fibrosis known as asbestosis as well as cancers such as malignant mesothelioma and lung cancer. Asbestos is a mineral silicate containing iron, magnesium, and calcium with a core of $\mathrm{SiO}_{2}$. The immunological effect of silica, $\mathrm{SiO}_{2}$, involves the dysregulation of autoimmunity because of the complications of autoimmune diseases found in silicosis. Asbestos can therefore cause alteration of immunocompetent cells to result in a decline of tumor immunity. Additionally, due to its physical characteristics, asbestos fibers remain in the lung, regional lymph nodes, and the pleural cavity, particularly at the opening sites of lymphatic vessels. Asbestos can induce chronic inflammation in these areas due to the production of reactive oxygen/nitrogen species. As a consequence, immunocompetent cells can have their cellular and molecular features altered by chronic and recurrent encounters with asbestos fibers, and there may be modification by the surrounding inflammation, all of which eventually lead to decreased tumor immunity. In this paper, the brief results of our investigation regarding reduction of tumor immunity of immunocompetent cells exposed to asbestos in vitro are discussed, as are our findings concerned with an investigation of chronic inflammation and analyses of peripheral blood samples derived from patients with pleural plaque and mesothelioma that have been exposed to asbestos.
\end{abstract}

\section{Introduction}

Asbestos causes lung fibrosis known as asbestosis, a few benign pleural diseases such as pleural plaque and effusion, and malignant diseases such as mesothelioma and lung cancers [1-5]. Furthermore, cancers in other organs such as the gastrointestinal tract, larynx, kidney, liver, pancreas, ovary, and hematopoietic systems show ahigh prevalence in asbestosinhaled people [6-9]. This issue has been tackled as a major medical and social problem throughout the world, especially since asbestos is very useful in various industries for its mineral characteristics such as resistance to heat, cold, chemicals, cheapness, easiness to obtain and weave, and so on $[8,9]$.

In Japan, the quantity of asbestos produced has increased since the mid-1950s and reached a peak in 1974 (over $350,000 \mathrm{z}$ tons) [10-12]. The peak level of usage continued until the late 1980s in construction, car, and other industries. In the summer of 2005, Kubota Corporation, which mainly 
used asbestos to make water pipes in Amagasaki City, Hyogo Prefecture, Japan, acknowledged the prevalence of asbestosrelated diseases among their workers, including several patients living near Kubota's asbestos-handling factory in Amagasaki City [10-12]. Citizens in Japan were suddenly made aware that asbestos causes malignancies in asbestos-handling workers and in residents living near these factories. They were informed that mesothelioma is difficult to diagnose and cure and were angered that workers and neighborhood residents had not been notified that these factories had been handling this silent bomb, asbestos [10-12].

To reduce the anxieties of the Japanese people, epidemiological analyses commenced regarding the Amagasaki area, and clinical and basic research was conducted on the biological effects of asbestos and the early detection of mesothelioma. It is in this context that the authors became involved in the project "Comprehensive Approach on Asbestos-Related Diseases", supported by the "Special Coordination Funds for Promoting Science and Technology" (headed by Dr. Takemi Otsuki, Department of Hygiene, Kawasaki Medical School, Kurashiki, Japan) from 2006 to 2010. In this project, a case and clinical specimen registration system was established. A feasible clinical trial was established and involved a combined trimodality therapy using anticancer chemotherapy with cisplatin and pemetrexed, followed by extrapleural pneumonectomy and postoperative radiation therapy for early-stage mesothelioma patients $[13,14]$. Furthermore, early detection procedures were developed using serum or pleural effusions to measure soluble mesothelin-related peptide (SMRP) and other markers such as osteopontin, vascular endothelial growth Factor (VEGF), and angiopoietin-1 as well as procedures for detection of circulating mesothelioma cells and circulating epithelial cells using peripheral blood [15-19].

For the basic research, the project "Comprehensive Approach on Asbestos-Related Diseases" included three subgroups: (1) analyses of cellular and molecular characteristics using mesothelioma cell lines, (2) an investigation of asbestos-induced carcinogenesis using an animal model, and (3) a study of the immunological effects of silica/asbestos.

The basic research project (3) was performed by us, and in this paper, we introduce our viewpoint that asbestos may cause alteration of immunocompetent cells resulting in chronic inflammation as well as tumor development.

\section{Immunological Effects of the Mineral Silicate on Asbestos and Silica}

Asbestos is a mineral silicate containing iron, magnesium, and calcium, with a core of "Si" and "O" $[6,7]$. Individuals exposed to silica and asbestos develop lung fibrosis known as silicosis and asbestosis, respectively $[1-5,20]$. Silicosis is a form of occupational lung disease caused by inhalation of crystalline silica dust, and is marked by inflammation and scarring in the form of nodular lesions in the upper lobes of the lungs [20]. However, asbestosis developsin patients who inhale a relatively high dose of asbestos (compared with patients with pleural lesions such as mesothelioma and plaque). In addition, the pathology of asbestosis differs from that of silicosis [1-5]. Asbestosis involves the scarring of lung tissue (around the terminal bronchioles and alveolar ducts). There are two types of fibers: amphibole (thin and straight) and serpentine (curved). The former are primarily responsible for human disease as they are able to penetrate deeply into the lungs. When such fibers reach the alveoli in the lung, where oxygen is transferred into the blood, the foreign bodies (asbestos fibers) cause the activation of the lung's local immune system and provoke an inflammatory reaction [1-5]. This inflammatory reaction can be described as chronic rather than acute, slow ongoing activation of the immune system in an attempt to eliminate the foreign fibers. Macrophages phagocytose the fibers and stimulate fibroblasts to deposit connective tissue. Due to the natural resistance of asbestos fibers to digestion, the macrophage dies off, releasing cytokines and attracting further lung macrophages and fibroblastic cells to lay down fibrous tissue, which eventually forms a fibrous mass $[21,22]$. The result is interstitial fibrosis. The fibrotic scar tissue causes alveolar walls to thicken, which reduces elasticity and gas diffusion, reducing oxygen transfer to blood as well as the removing of carbon dioxide.

Furthermore, the complications in silicosis and in people who have inhaled asbestos are also different. Patients with silicosis often present with complications involving autoimmune diseases such as rheumatoid arthritis (known as Caplan's syndrome) [23-25], systemic sclerosis [26, 27], systemic lupus erythematosus [28, 29], and antineutrophil cytoplasmic antibody (ANCA)-related vasculitis/nephritis [30-32]. Although these have been considered adjuvant effects of silica, we have assumed that silica may influence circulating immunocompetent cells, particularly T lymphocytes, and have reported that silica can activate CD4 + 25 + FoxP3 (forkhead box P3) + regulatory $\mathrm{T}$ cells (Treg) and responder $\mathrm{T}$ cells (Tresp) [33-38]. These activations induce overexpression of CD95/Fas in Treg, resulting in early loss and contamination of activated Tresp, which express CD25 as the marker for activation into a peripheral $\mathrm{CD} 4+25+$ subpopulation of $\mathrm{T}$ cells. These phenomena seem to induce reduced regulation of autoimmunity [37, 38]. Regarding silica-induced fibrosis (lung and skin), the idea proposed previously [27] is that silica affect alveolar macrophages, endothelial cells, and fibroblasts to modify cytokine production and disturbance of collagen synthesis and degradation, subsequently forming fibrosis of lung and skin lesion. In addition, our findings suggested that silica may influence and alter circulating lymphocytes to disturb autoimmune tolerance $[37,38]$.

On the other hand, a consideration of the complications of asbestos exposure indicates that the development of cancers is the most important aspect [1-5]. As silica can affect immunocompetent cells, asbestos may possess a similar influence on various immune cells, and the results should be the reduction of tumor immunity. As mentioned above, in addition to lung cancer and mesothelioma, there may be a relatively high prevalence of other cancers among asbestosinhaled patients $[6,7]$.

We have reported that the natural killer (NK) cell line and freshly isolated NK cells derived from healthy donors (HDs) exposed to asbestos (chrysotile) for a long period (more than half a year in vitro for the cell line and approximately two 
weeks for the fresh NK cells) showed a reduction of cytotoxicity with decreased expression of their activating receptors, such as NKG2D and 2B4 in the cell line and NKp46 in the fresh NK cells [39]. The NK cell line exposed to asbestos also showed suppressed signaling such as extracellular signalregulated kinas (ERK) 1/2 in the mitogen-activated protein kinase (MAPK) cascade from activating receptors and also producting of granzyme and perforin $[40,41]$. At present, the effects of chrysotile on CD8+ cytotoxic T lymphocytes (CTL) are also being analyzed, and findings show reduction of differentiation from naïve CTL to effector/memory CTL and proliferation [42].

\section{Chronic Inflammation and Development of Mesothelioma}

Inhaled asbestos is usually handled by alveolar macrophages. Recently, the role of a NOD-like receptor family, the pyrin domain containing 3 (NLRP3) (NACHT, LRR, and PYD domains-containing protein 3; Nalp3) inflammasome, has received attention regarding the handling of these foreign bodies as well as various crystalline substances such as uric acid and cholesterol crystalline causing atherosclerosis [4347]. The following cellular and molecular events then occur. (1) Capture of silica/asbestos by macrophages and entrapment within lysosomes. (2) Activation of NLRP3 inflammasome to cleave procaspase 1 to an active form. (3) Cleavage of prointerleukin (IL)-1 $\beta$ to an active form for release to form fibrotic nodules. (4) Production of reactive oxygen species (ROS) and reactive nitrogen species (RNS) in the macrophages. (5) Induction of cellular and tissue damage due to the production of ROS and RNS. (6) Apoptosis of the alveolar macrophages. (7) Production of various cytokines/chemokines such as IL- $1 \beta$ tumor necrosis factor (TNF)- $\alpha$, macrophage inflammatory protein (MIP)-1/2, monocyte-chemoattractant protein-1, and IL-8 to cause chronic inflammation and proliferation of collagenic fibers. (8) Release of silica particles and asbestos fibers from alveolar macrophages and the repetition of similar cellular reactions described above by newly recognized nearby macrophages. (9) Transfer of silica particles and (partially cleaved) asbestos fibers to regional lymph nodes. (10) As these cellular and molecular reactions are continuously repeated, pulmonary fibrosis will appear gradually and progressively $[48,49]$.

As a result of these cellular and molecular events, cleaved asbestos fibers will accumulate in regional lymphonodes, the distal end of the alveolus, and the pleural cavity, particularly at the opening of lymphatic vessels (Figure 1) [1-5]. Circulating and local immunocompetent cells may encounter asbestos fibers repeatedly, recurrently, and continuously.

Asbestos, particularly amphibole in the form of crocidolite and amosite, includes iron and is considered responsible for the production of reactive oxygen and nitrogen species (ROS and RNS) that may cause DNA damage to nearby cells and induce the development of cancers [50-52]. In addition, these ROS and RNS may develop local chronic inflammation. It has been thought that chronic inflamma- tion contributes to a substantial part of environmental carcinogenesis [53-55]. Various infectious diseases and physi$\mathrm{cal}$, chemical, and immunological factors participate in inflammation-related carcinogenesis [50-55]. For example, hepatocellular carcinoma, cervical cancer of uterine, and bladder cancer are known to be caused by infection of hepatitis $\mathrm{C}$ virus, human papilloma virus (HPV), and Schistosoma haematobium, respectively. In addition, Helicobacter pylori infection causes gastric cancer, extranodal marginal zone lymphoma of mucosa-associated lymphoid tissue type (MALT lymphoma), and diffuse large B-cell lymphoma. Regarding incidences of these diseases, WHO reported about Hepatitis $C$ virus [56]. As many as 2 to 4 million persons may be chronically infected in the United States, 5 to 10 million in Europe, and about 12 million in India, and most do not know that they are infected. About 150,000 new cases occur annually in the USA and in western Europe and about 350,000 in Japan. Of these, about $25 \%$ are symptomatic, but 60 to $80 \%$ may progress to chronic liver disease, and $20 \%$ of these develop cirrhosis. About $5 \%-7 \%$ of patients may ultimately die of the consequences of the infection. In addition, HPV causes cervical cancer which is the second most common cancer in women worldwide. In 2008 , there were an estimated 529,000 new cases and 274,00 deaths due to cervical cancer [57]. It was reported that 8-nitroguanine was formed at sites of carcinogenesis in animal models and patients with various cancer-prone infectious and inflammatory diseases, caused by parasites, viruses, and asbestos exposure. In asbestos-exposed mice, 8-nitroguanine was formed in bronchial epithelial cells, and it is noteworthy that crocidolite induced significantly more intense 8-nitroguanine formation than chrysotile, findings that are inconsistent with their respective carcinogenic potentials [58].

From the above-mentioned basic research (2) concerning "investigation of asbestos-induced carcinogenesis using an animal model" in the "Comprehensive Approach on Asbestos-Related Diseases" project, the importance of iron is supposed even in the development of mesothelioma caused by iron-free chrysotile because of its easy binding to hemoglobin and the induced hemolysis [59-61]. Immunocompetent cells may show some alteration as characteristics of chronic inflammation and features involving reduced tumor immunity.

From this viewpoint, even though we do not observe cellular and molecular changes for immunocompetent cells, we reported interesting findings regarding the reduction of tumor immunity in $\mathrm{T}$ cells. We then introduce the findings concerning reduction of CXC chemokine receptor (CXCR) 3 and interferon (IFN) $-\gamma$ with the activated potential expression of IL-6.

\section{Chemokine Receptor CXCR3 Expression and Its Relation To Interferon (IFN)- $\gamma$ and IL-6}

Similar to analyses of NK cells, we adopted a human T-cell line, MT-2, as the chronic and continuous exposure model of $\mathrm{T}$ cells. Although the altered features of continuously exposed (to chrysotile) sublines (we established six sublines 


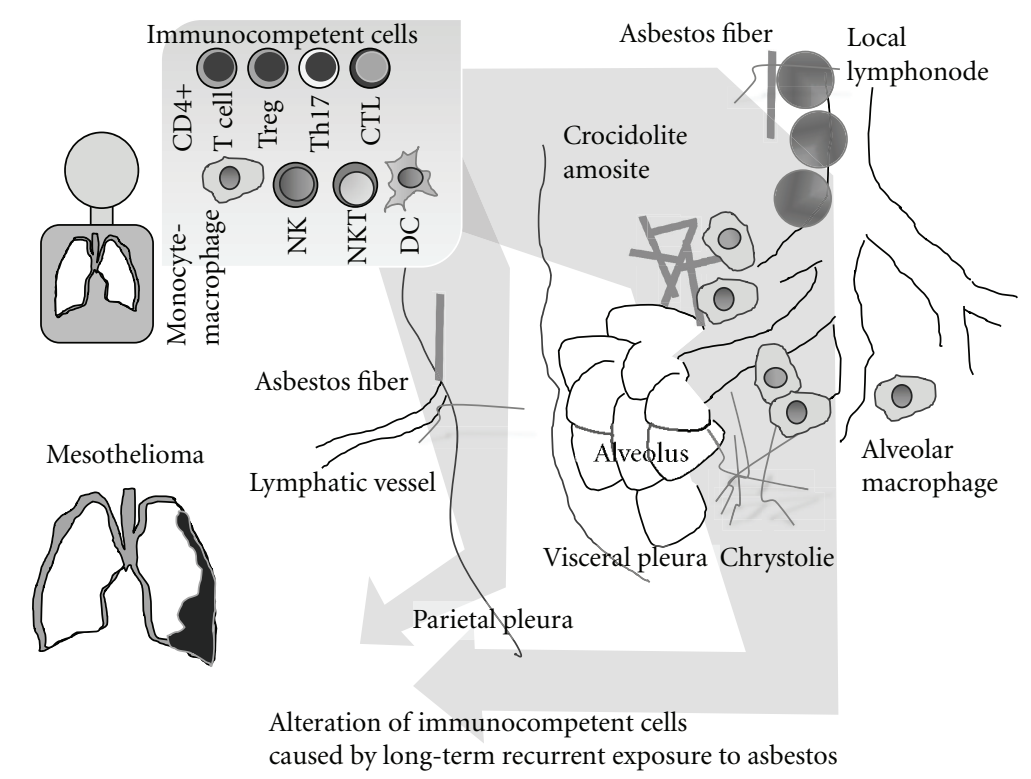

FIGURE 1: Localization of inhaled asbestos fibers in the lung, pleural cavity, and regional lymphonodes. Chronic and recurrent encounters between circulating immunocompetent cells and these moored asbestos fibers causing the long-term alteration of these cells.

independently exposed to chrysotile) of MT-2 were reported previously [62-64], one of the interesting molecular changes regarding tumor immunity is the reduction of CXCR3 expression and IFN- $\gamma$ production [65-68]. CXCR3 is thought to be important for inflammation, since CXCR3 is known as the receptor for CXCL9, 10, and 11 which induce inflammation. In addition, CXCR3 expressing $\mathrm{T}$ cells in the tumorlocalized region recruit IFN- $\gamma$-producing cells to kill the tumor cells.

Results using the MT-2 cell line model, as shown in the left upper panel of Figure 2, indicated that continuously exposed sublines of MT-2 showed reduced CXCR3 expression on their surface and mRNA expression levels, with reduced production and expression of IFN- $\gamma$. Production of the Th1 type CXCR3 ligand CXCL10/IP10 was also significantly reduced in sublines compared with the original line. In addition, another Th1-type chemokine, CCL4/MIP- $1 \beta$ mRNA, was also expressed at low levels in all six sublines compared with the MT-2 original line as previously reported $[69,70]$. However, CCR5, the Th1-type receptor for CCL4/MIP-1 $\beta$, was not reduced significantly through mRNA expression in MT-2Rsts cells. These results indicated that continuous exposure of MT-2 original cells to asbestos altered the expression of Th1-related chemokines (CXCL10/IP10 and CCL4/MIP-1 $\beta$ ) and chemokine receptors (CXCR3) $[69,70]$.

Similar to analyses regarding the effects of asbestos on NK cells, we then tried to determine whether freshly isolated human peripheral CD4+ $\mathrm{T}$ cells show a similar alteration ex vivo when proliferation is maintained by IL-2-containing medium in the presence of chrysotile as shown in the left lower panel of Figure $2[69,70]$. After several weeks of coculture supplemented with IL-2 in the presence or absence of chrysotile, cell surface CXCR3 expression decreased in a dose-dependent manner. Thus, we examined cell surface ex- pression of CXCR3 and CCR5 in CD4+ T cells derived from six healthy donors, since both receptors are preferentially expressed in Th1/effect or T cells. The expression of CXCR3 was significantly reduced following exposure to $10 \mu \mathrm{g} / \mathrm{mL}$ of chrysotile for 28 days, although this difference seemed to depend on one case in which the expression decreased remarkably [69, 70]. Even if the culture conditions for the CD4+ T cells was limited to a period of around four weeks, four of the six HDs showed a decrease of CXCR3 expression to various degrees, and it might be concluded that asbestos exposure potentiates reduction of CXCR3 expression in CD4+ T cells. These results indicated that CXCR3 expression might be specifically reduced by asbestos exposure. In addition, these experiments revealed decreased IFN- $\gamma$ expression and production when CD4+ T cells from HDs were cultured with chrysotile for 28 days $[69,70]$.

Finally, analyses of changes in surface CXCR3 expression on freshly isolated CD4+ T cells from asbestos-exposed patients such as those with pleural plaque (PP) or malignant mesothelioma (MM) were compared with those from HDs. In addition, IFN- $\gamma$ and IL- 6 expression of CD4+ T cells from these patients and HDs was measured with stimulation using anti-CD3/CD28 antibodies with IL-2 [69, 70]. As summarized in the right panel of Figure 2, CXCR3 expression was reduced in CD4+ T cells from asbestos-exposed patients. A comparison of PP and MM patients showed that the expression level of CXCR3 on CD4+ T cells from MM was decreased, although the difference was not statistically significant. Moreover, although IFN- $\gamma$ expression was only reduced in stimulated CD4+ T cells from MM patients and not in those with PP, IL-6 expression was gradually enhanced in HDs and to a lesser extent in PP, followed by MM. As we reported previously, the plasma level of IL-6 was significantly higher in MM compared to HDs, PP, and silicosis [71]. 


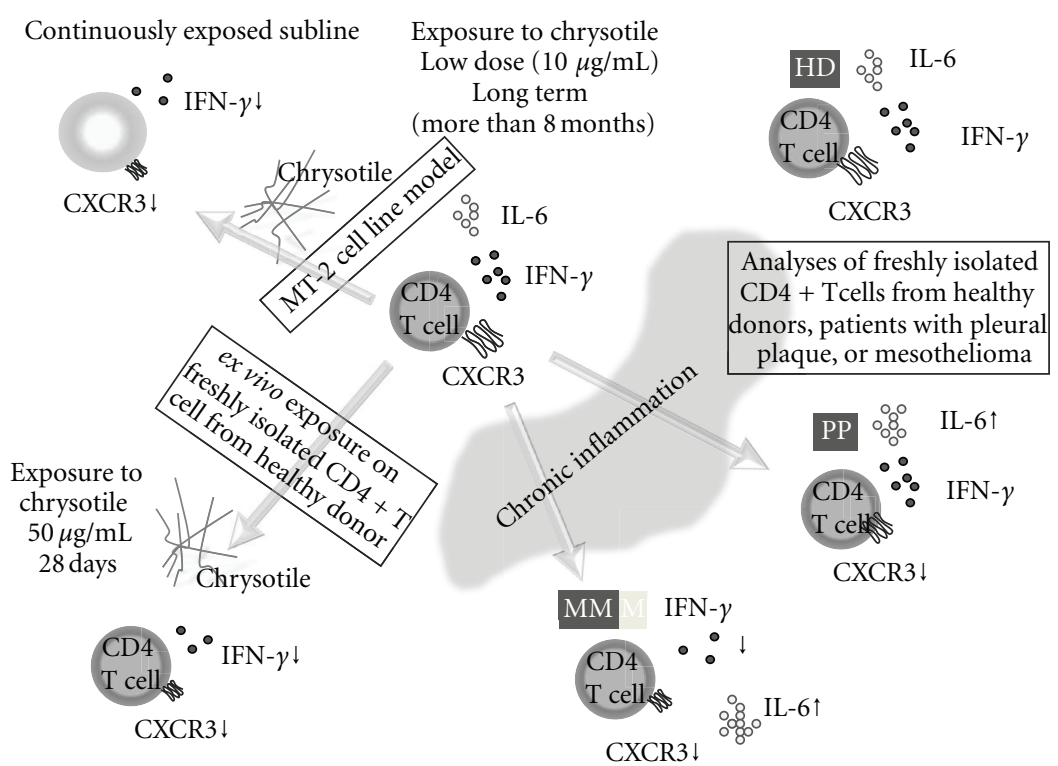

FIGURE 2: Schematic representation of asbestos-induced reduction of expression of a chemokine receptor, CXCR3, and expression and production of IFN- $\gamma$ with increasing IL-6 using the MT-2 cell line model exposed continuously to a low dose of chrysotile (upper left), an ex vivo exposure model using freshly isolated CD4+ T cells from healthy donors (HD) (lower left) as well as analyses of freshly isolated CD4+ T cells from healthy donors and patients with pleural plaque (PP) and malignant mesothelioma (MM) (right).

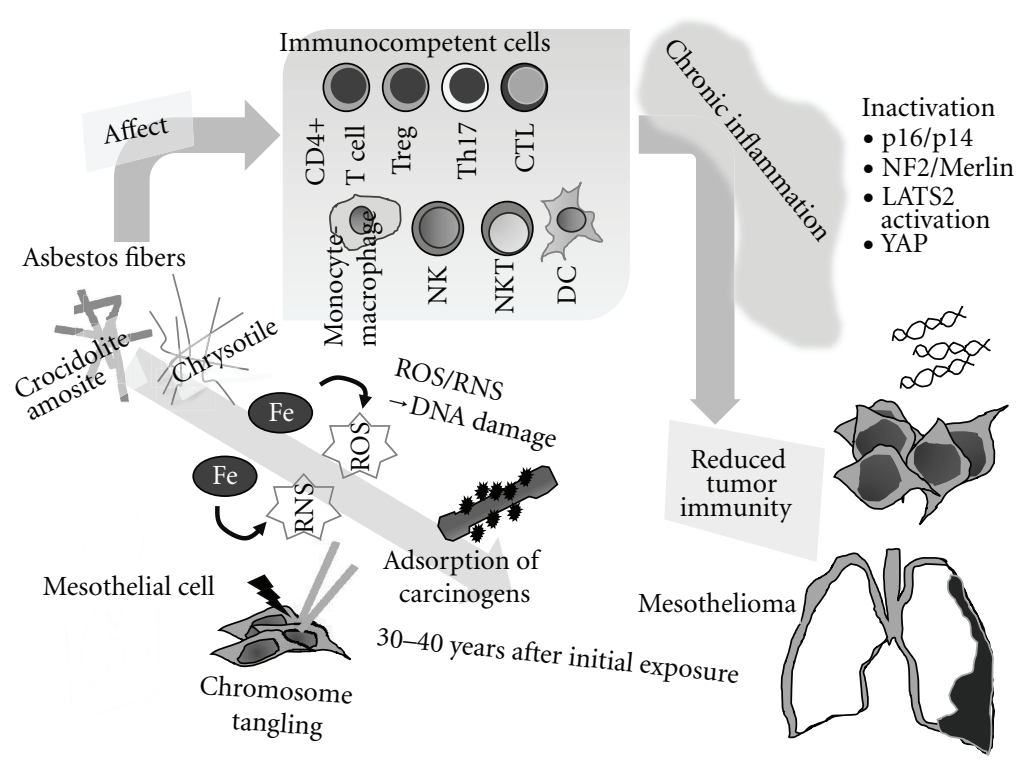

FIGURE 3: Schematic model showing mechanisms of asbestos-induced carcinogenesis and genomic/epigenetic changes found in mesothelioma cells, carcinogenic activities of asbestos fibers, and the relationship of the immunological effects of asbestos in regard to chronic in-flammation and reduced tumor immunity.

Although this may depend on the tumor-producing IL-6 [72-74], our findings indicated that part of the increased IL-6 may be produced by T cells with altered potentials to express cytokines due to continuous exposure to asbestos. Furthermore, IL-6 is an interleukin that acts as a proinflammatory and anti-inflammatory cytokine. It is secreted by $\mathrm{T}$ cells and macrophages to stimulate an immune response, for example, during infection and after trauma, especially in the case of burns or other tissue damage leading to inflam- mation [75-78]. For these reasons, it can be assumed that immunocompetent cells possess cellular characteristics of chronic inflammatory alterations during continuous exposure to asbestos, and they then proceed to result in the reduction of tumor immunity as shown in Figure 3. In addition, the supposed difference of immunological effects between silica and asbestos is shown in Figure 4. However, although there is insufficient evidence for all of these sequential modifications of immunocompetent cells, 


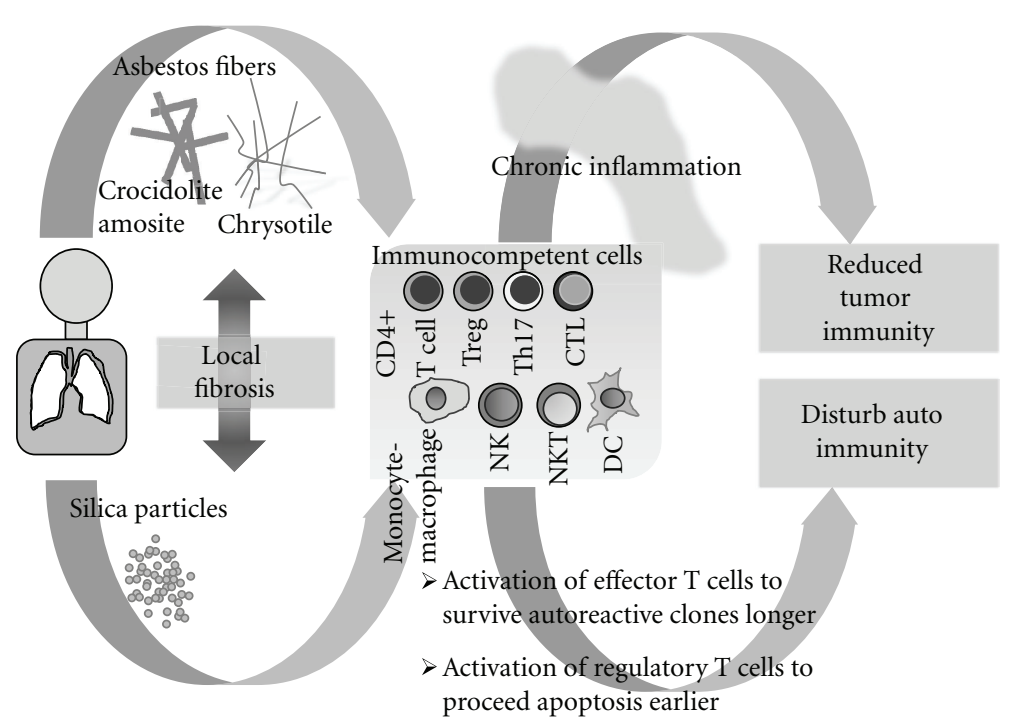

FIGURE 4: Schematic model presenting the differences of immunological effects of silica and asbestos based on our findings. Silica may disturb the autoimmunity, and asbestos may cause reduction of tumor immunity via forming chronic inflammation.

an investigation of their long-term alteration may lead to the development of preventive tools for asbestos-induced malignancies. For example, it may be possible to find some physiologically active substances in the plants or microorganisms to modify or recover the altered function of immunocompetent cells to reconstitute the tumor immunity in asbestosexposed people, and discriminate iron from bodies exposed to asbesots.

\section{Conclusion}

As shown in Figure 3, the carcinogenic activity of asbestos encompasses the following phenomena: (1) DNA damage caused by ROS/RNS production due to the iron presentin asbestos fibers, (2) chromosome tangling to result in DNA damage due to the physical features of asbestos fibers, and (3) adsorption of various carcinogensaround the asbestos fibers [59-61]. In addition, the molecular events regarding carcinogenesis found in mesothelioma cells include (1) homogenous deletion of $p 16^{I N K 4 a} / p 19^{A R F}$ found in more than $90 \%$ of cases, (2) inactivation of neurofibromatosis 2 (NF2)/Merlin found in approximately half of the cases, (3) inactivation of the serine/threonine-protein kinase (LATS2) gene in approximately one-third of mesothelioma cell lines and representinga candidate for a novel tumor suppressor in $\mathrm{MM}$, and (4) transcription factor, Yes-associated protein (YAP) involved in the NF2/Merlin-hippo signaling pathway and constitutively dephosphorylated by LATS, and usually acting as an oncogene to bind with the TEAD transcription factor to enhance the cell cycle and resistance to apoptosis [79-81].

Asbestos fibers and the cellular and molecular characteristics of mesothelioma cells may lead to the gradual alteration of immunocompetent cells and subsequent development of chronic inflammation and later reduction of tumor immunity. Investigation of the progression of modification in im- munocompetent cells caused by exposure to asbestos may lead to the development of novel methods for the prevention of mesothelioma and other asbestos-related cancers.

\section{Acknowledgments}

The authors specially thank Ms. Yoshiko Yamashita, Minako Kato, Tomoko Sueishi, Keiko Kimura, Misao Kuroki, and Naomi Miyahara for their technical help. The experimental results performed by them and presented partly in this paper were supported by Special Funds for Promoting Science and Technology (H18-1-3-3-1), JSPS KAKENHI (22790550, 22700933, 20390178, 20890270, 19689153, 19790431, 19790411, 18390186, 16390175, and 09670500), the Takeda Science Foundation (Tokutei Kenkyu Josei I, 2008), and Kawasaki Medical School Project, Grants (22-B1, 22-A58, 22-A29, 21-401, 21-201, 21-107, 20-411I, 20-210O, 20-109N, 20-402O, and 20-410I).

\section{References}

[1] J. E. Craighead and A. R. Gibbs, Asbestos and Its Diseases, Oxford University Press, New York, NY, USA, 2008.

[2] R. F. Dodson and S. P. Hammar, Asbestos: Risk Assessment, Epidemiology, and Health Effects, CRC Press, Boca Ratton, Fla, USA, 2006.

[3] V. L. Roggli, T. D. Oury, and T. A. Sporn, Springer Science and Business Media, Springer Science and Business Media, New York, NY, USA, 2nd edition, 2004.

[4] K. O'Byrne and V. Rusch, Malignant Pleural Mesothelioma, Oxford University Press, New York, NY, USA, 2006.

[5] A. Baldi, Nova Scientific, Nova Scientific Publishers, New York, NY, USA, 2008.

[6] J. E. Craighead, "Nonthoracic cancers possibly resulting from asbestos exposure," in Asbestos and Its Diseases, J. E. Craighead and A. R. Gibbs, Eds., pp. 230-252, Oxford University Press, New York, NY, USA, 2008. 
[7] R. Rolston and D. Oury, "Other neoplasia," in Pathology of Asbestos-Associated Diseases, V. L. Roggli, T. D. Oury, and T. A. Sporn, Eds., pp. 217-230, Springer Science and Business Media, New York, NY, USA, 2nd edition, 2004.

[8] J. E. Craighead, Al. Gibbs, and F. Pooley, "Mineralogy of asbestos," in Asbestos and Its Diseases, J. E. Craighead and A. R. Gibbs, Eds., pp. 23-38, Oxford University Press, New York, NY, USA, 2008.

[9] V. L. Roggli and P. Coin, "Mineralogy of asbestos," in Pathology of Asbestos-Associated Diseases, V. L. Roggli, T. D. Oury, and T. A. Sporn, Eds., pp. 1-16, Springer Science and Business Media, New York, NY, USA, 2nd edition, 2004.

[10] N. Kurumatani and S. Kumagai, "Mapping the risk of mesothelioma due to neighborhood asbestos exposure," American Journal of Respiratory and Critical Care Medicine, vol. 178, no. 6, pp. 624-629, 2008.

[11] S. Kumagai and N. Kurumatani, "Asbestos fiber concentration in the area surrounding a former asbestos cement plant and excess mesothelioma deaths in residents," American Journal of Industrial Medicine, vol. 52, no. 10, pp. 790-798, 2009.

[12] S. Kumagai, N. Kurumatani, T. Tsuda, T. Yorifuji, and E. Suzuki, "Increased risk of lung cancer mortality among residents near an asbestos product manufacturing plant," International Journal of Occupational and Environmental Health, vol. 16, no. 3, pp. 268-278, 2010.

[13] T. Yamanaka, F. Tanaka, S. Hasegawa et al., "A feasibility study of induction pemetrexed plus cisplatin followed by extrapleural pneumonectomy and postoperative hemithoracic radiation for malignant pleural mesothelioma," Japanese Journal of Clinical Oncology, vol. 39, no. 3, pp. 186-188, 2009.

[14] S. Hasegawa and F. Tanaka, "Malignant mesothelioma: current status and perspective in Japan and the world," General Thoracic and Cardiovascular Surgery, vol. 56, no. 7, pp. 317-323, 2008.

[15] R. Ohashi, K. Tajima, F. Takahashi et al., "Osteopontin modulates malignant pleural mesothelioma cell functions in vitro," Anticancer Research, vol. 29, no. 6, pp. 2205-2214, 2009.

[16] C. Tabata, N. Hirayama, R. Tabata et al., "A novel clinical role for angiopoietin-1 in malignant pleural mesothelioma," European Respiratory Journal, vol. 36, no. 5, pp. 1099-1105, 2010.

[17] A. Yasumitsu, C. Tabata, R. Tabata et al., "Clinical significance of serum vascular endothelial growth factor in malignant pleural mesothelioma," Journal of Thoracic Oncology, vol. 5, no. 4, pp. 479-483, 2010.

[18] Y. Okumura, F. Tanaka, K. Yoneda et al., "Circulating tumor cells in pulmonary venous blood of primary lung cancer patients," Annals of Thoracic Surgery, vol. 87, no. 6, pp. 1669$1675,2009$.

[19] F. Tanaka, K. Yoneda, N. Kondo et al., "Circulating tumor cell as a diagnostic marker in primary lung cancer," Clinical Cancer Research, vol. 15, no. 22, pp. 6980-6986, 2009.

[20] IARC ,International Agency for Research on Cancer, World Health Organization, "Silica, some silicate, coal dust and paraaramid fibrils," IARC Monographs on the Evaluation of Carcinogenic Risks to Humans, vol. 68, 1997.

[21] N. H. Heintz and B. T. Mossman, "Molecular responses to asbestos: induction of cell proliferation and apoptosis through modulation of redox-dependent cell signaling pathway," in Asbestos and Its Diseases, J. E. Craighead and A. R. Gibbs, Eds., pp. 120-138, Oxford University Press, New York, NY, USA, 2008.

[22] M. A. L. Atkinson, "Molecular and cellular responses to asbestos exposure," in Asbestos: Risk Assessment, Epidemiology, and
Health Effects, R. F. Dodson and S. P. Hammar, Eds., pp. 39-90, CRC Press, Boca Ratton, Fla, USA, 2006.

[23] A. Caplan, "Rheumatoid disease and pneumoconiosis (Caplan's syndrome)," Proceedings of the Royal Society of Medicine, vol. 52, pp. 1111-1113, 1959.

[24] A. Caplan, R. B. Payne, and J. L. Withey, "A broader concept of Caplan's syndrome related to rheumatoid factors," Thorax, vol. 17, pp. 205-212, 1962.

[25] J. Schreiber, D. Koschel, J. Kekow, N. Waldburg, A. Goette, and R. Merget, "Rheumatoid pneumoconiosis (Caplan's syndrome)," European Journal of Internal Medicine, vol. 21, no. 3, pp. 168-172, 2010.

[26] K. M. Reiser and J. A. Last, "Silicosis and fibrogenesis: fact and artifact," Toxicology, vol. 13, no. 1, pp. 51-72, 1979.

[27] U. F. Haustein and U. Anderegg, "Silica induced scleroderma-Clinical and experimental aspects," Journal of Rheumatology, vol. 25, no. 10, pp. 1917-1926, 1998.

[28] C. L. Uber and R. A. McReynolds, "Immunotoxicology of silica," Critical Reviews in Toxicology, vol. 10, no. 4, pp. 303320, 1982.

[29] K. Steenland and D. F. Goldsmith, "Silica exposure and autoimmune diseases," American Journal of Industrial Medicine, vol. 28, no. 5, pp. 603-608, 1995.

[30] J. W. Cohen Tervaert, C. A. Stegeman, and C. G. M. Kallenberg, "Silicon exposure and vasculitis," Current Opinion in Rheumatology, vol. 10, no. 1, pp. 12-17, 1998.

[31] Z. Rihova, D. Maixnerova, E. Jancova et al., "Silica and asbestos exposure in ANCA-associated vasculitis with pulmonary involvement," Renal Failure, vol. 27, no. 5, pp. 605-608, 2005.

[32] J. Bartuňková, D. Pelclová, Z. Fenclová et al., "Exposure to silica and risk of ANCA-associated vasculitis," American Journal of Industrial Medicine, vol. 49, no. 7, pp. 569-576, 2006.

[33] P. Wu, Y. Miura, F. Hyodoh et al., "Reduced function of CD4+25+ regulatory $\mathrm{T}$ cell fraction in silicosis patients," International Journal of Immunopathology and Pharmacology, vol. 19, no. 2, pp. 357-368, 2006.

[34] H. Hayashi, M. Maeda, S. Murakami et al., "Soluble interleukin-2 receptor as an indicator of immunological disturbance found in silicosis patients," International Journal of Immunopathology and Pharmacology, vol. 22, no. 1, pp. 53-62, 2009.

[35] H. Hayashi, Y. Miura, M. Maeda et al., "Reductive alteration of the regulatory function of the CD4(+)CD25(+) T cell fraction in silicosis patients," International Journal of Immunopathology and Pharmacology, vol. 23, no. 4, pp. 1099-1109, 2010.

[36] H. Hayashi, Y. Nishimura, F. Hyodo et al., "Dysregulation of autoimmunity caused by silica exposure: fas-mediated apoptosis in T lymphocytes derived from silicosis patients," in Autoimmune Disorders: Symptoms, Diagnosis and Treatment, M. E. Petro, Ed., pp. 293-301, Nova Science Publishers, Hauppauge, NY, USA, 2011.

[37] N. Kumagai, H. Hayashi, M. Maeda et al., "Immunological effects of silica and related dysregulation of autoimmunity," in Autoimmune Disorder, C. Mavragani, Ed., vol. 1, InTech Open Access Publisher, Croatia.

[38] T. Otsuki, H. Hayashi, Y. Nishimura et al., "Dysregulation of autoimmunity caused by silica exposure and alteration of Fasmediated apoptosis in T lymphocytes derived from silicosis patients," International Journal of Immunopathology and Pharmacology, vol. 24, no. 1, supplement, pp. 11S-16S, 2011.

[39] Y. Nishimura, Y. Miura, M. Maeda et al., "Impairment in cytotoxicity and expression of NK cell-activating receptors on human NK cells following exposure to asbestos fibers," International Journal of Immunopathology and Pharmacology, vol. 22, no. 3, pp. 579-590, 2009. 
[40] Y. Nishimura, M. Maeda, N. Kumagai, H. Hayashi, Y. Miura, and T. Otsuki, "Decrease in phosphorylation of ERK following decreased expression of NK cell-activating receptors in human NK cell line exposed to asbestos," International Journal of Immunopathology and Pharmacology, vol. 22, no. 4, pp. 879-888, 2009.

[41] Y. Nishimura, N. Kumagai, M. Maeda et al., "Suppressive effect of asbestos on cytotoxicity of human NK cells," International Journal of Immunopathology and Pharmacology, vol. 24, no. 1, supplement, pp. 5S-10S, 2011.

[42] M. Maeda, Y. Nishimura, N. Kumagai et al., "Dysregulation of the immune system caused by silica and asbestos," Journal of Immunotoxicology, vol. 7, no. 4, pp. 268-278, 2010.

[43] C. Dostert, V. Pétrilli, R. Van Bruggen, C. Steele, B. T. Mossman, and J. Tschopp, "Innate immune activation through Nalp3 inflammasome sensing of asbestos and silica," Science, vol. 320, no. 5876, pp. 674-677, 2008.

[44] S. L. Cassel, S. C. Eisenbarth, S. S. Iyer et al., "The Nalp3 inflammasome is essential for the development of silicosis," Proceedings of the National Academy of Sciences of the United States of America, vol. 105, no. 26, pp. 9035-9040, 2008.

[45] S. Benko, D. J. Philpott, and S. E. Girardin, "The microbial and danger signals that activate Nod-like receptors," Cytokine, vol. 43, no. 3, pp. 368-373, 2008.

[46] F. Martinon and J. Tschopp, "Inflammatory caspases: linking an intracellular innate immune system to autoinflammatory diseases," Cell, vol. 117, no. 5, pp. 561-574, 2004.

[47] K. Schroder and J. Tschopp, “The Inflammasomes," Cell, vol. 140, no. 6, pp. 821-832, 2010.

[48] R. F. Hamilton, S. A. Thakur, and A. Holian, "Silica binding and toxicity in alveolar macrophages," Free Radical Biology and Medicine, vol. 44, no. 7, pp. 1246-1258, 2008.

[49] S. A. Thakur, R. F. Hamilton, and A. Holian, "Role of scavenger receptor A family in lung inflammation from exposure to environmental particles," Journal of Immunotoxicology, vol. 5, no. 2, pp. 151-157, 2008.

[50] D. Upadhyay and D. W. Kamp, "Asbestos-induced pulmonary toxicity: role of DNA damage and apoptosis," Experimental Biology and Medicine, vol. 228, no. 6, pp. 650-659, 2003.

[51] K. Bhattacharya, E. Dopp, P. Kakkar et al., "Biomarkers in risk assessment of asbestos exposure," Mutation Research, vol. 579, no. 1-2, pp. 6-21, 2005.

[52] G. Liu, R. Beri, A. Mueller, and D. W. Kamp, "Molecular mechanisms of asbestos-induced lung epithelial cell apoptosis," Chemico-Biological Interactions, vol. 188, no. 2, pp. 309$318,2010$.

[53] M. Philip, D. A. Rowley, and H. Schreiber, "Inflammation as a tumor promoter in cancer induction," Seminars in Cancer Bio$\log y$, vol. 14, no. 6, pp. 433-439, 2004.

[54] S. C. Robinson and L. M. Coussens, "Soluble mediators of inflammation during tumor development," Advances in Cancer Research, vol. 93, pp. 159-187, 2005.

[55] A. Federico, F. Morgillo, C. Tuccillo, F. Ciardiello, and C. Loguercio, "Chronic inflammation and oxidative stress in human carcinogenesis," International Journal of Cancer, vol. 121, no. 11, pp. 2381-2386, 2007.

[56] WHO website, "Hepatitis C," http://www.who.int/csr/disease/ hepatitis/whocdscsrlyo2003/en/index4.html\#incidence.

[57] WHO website, "New and under-utilized vaccines implementation (NUVI), human papillomavirus (HPV)," http://www. who.int/nuvi/hpv/en/.
[58] Y. Hiraku, S. Kawanishi, T. Ichinose, and M. Murata, "The role of iNOS-mediated DNA damage in infection-and asbestosinduced carcinogenesis," Annals of the New York Academy of Sciences, vol. 1203, pp. 15-22, 2010.

[59] S. Toyokuni, "Mechanisms of asbestos-induced carcinogenesis," Nagoya Journal of Medical Science, vol. 71, no. 1-2, pp. 110, 2009.

[60] S. Toyokuni, "Role of iron in carcinogenesis: cancer as a ferrotoxic disease," Cancer Science, vol. 100, no. 1, pp. 9-16, 2009.

[61] H. Nagai and S. Toyokuni, "Biopersistent fiber-induced inflammation and carcinogenesis: lessons learned from asbestos toward safety of fibrous nanomaterials," Archives of Biochemistry and Biophysics, vol. 502, no. 1, pp. 1-7, 2010.

[62] Y. Miura, Y. Nishimura, H. Katsuyama et al., "Involvement of IL-10 and Bcl-2 in resistance against an asbestos-induced apoptosis of T cells," Apoptosis, vol. 11, no. 10, pp. 1825-1835, 2006.

[63] T. Otsuki, M. Maeda, S. Murakami et al., "Immunological effects of silica and asbestos," Cellular \& Molecular Immunology, vol. 4, no. 4, pp. 261-268, 2007.

[64] M. Maeda, Y. Miura, Y. Nishimura et al., "Immunological changes in mesothelioma patients and their experimental detection," Clinical Medicine. Circulatory, Respiratory and Pulmonary, vol. 2, pp. 11-17, 2008.

[65] P. Romagnani, L. Lasagni, F. Annunziato, M. Serio, and S. Romagnani, "CXC chemokines: the regulatory link between inflammation and angiogenesis," Trends in Immunology, vol. 25, no. 4, pp. 201-209, 2004.

[66] U. P. Singh, C. Venkataraman, R. Singh, and J. W. Lillard, "CXCR3 axis: role in inflammatory bowel disease and its therapeutic implication," Endocrine, Metabolic and Immune Disorders, vol. 7, no. 2, pp. 111-123, 2007.

[67] A. M. Fulton, "The chemokine receptors CXCR4 and CXCR3 in cancer," Current Oncology Reports, vol. 11, no. 2, pp. 125131, 2009.

[68] S. Lacotte, S. Brun, S. Muller, and H. Dumortier, "CXCR3, inflammation, and autoimmune diseases," Annals of the New York Academy of Sciences, vol. 1173, pp. 310-317, 2009.

[69] M. Maeda, Y. Nishimura, H. Hayashi et al., "Decreased CXCR3 expression in CD4+ T cells exposed to asbestos or derived from asbestos-exposed patients," American Journal of Respiratory Cell and Molecular Biology, vol. 45, no. 4, pp. 795-803, 2011.

[70] M. Maeda, Y. Nishimura, H. Hayashi et al., "Reduction of CXCR3 in an in vitro model of continuous asbestos exposure on a human T-cell line, MT-2," American Journal of Respiratory Cell and Molecular Biology, vol. 45, no. 3, pp. 470-479, 2011.

[71] S. Murakami, Y. Nishimura, M. Maeda et al., "Cytokine alteration and speculated immunological pathophysiology in silicosis and asbestos-related diseases," Environmental Health and Preventive Medicine, vol. 14, no. 4, pp. 216-222, 2009.

[72] T. Motoyama, T. Honma, H. Watanabe, S. Honma, T. Kumanishi, and S. Abe, "Interleukin 6-producing malignant mesothelioma," Virchows Archiv B, vol. 64, no. 6, pp. 367-372, 1993.

[73] N. Kimura, T. Ogasawara, S. Asonuma, H. Hama, T. Sawai, and T. Toyota, "Granulocyte-colony stimulating factor-and interleukin 6-producing diffuse deciduoid peritoneal mesothelioma," Modern Pathology, vol. 18, no. 3, pp. 446-450, 2005.

[74] S. Eikawa, Y. Ohue, K. Kitaoka et al., "Enrichment of Foxp3+ CD4 regulatory $\mathrm{T}$ cells in migrated T cells to IL-6-and IL-8expressing tumors through predominant induction of CXCR1 
by IL-6," Journal of Immunology, vol. 185, no. 11, pp. 6734$6740,2010$.

[75] D. R. Hodge, E. M. Hurt, and W. L. Farrar, "The role of IL-6 and STAT3 in inflammation and cancer," European Journal of Cancer, vol. 41, no. 16, pp. 2502-2512, 2005.

[76] A. M. W. Petersen and B. K. Pedersen, "The role of IL-6 in mediating the anti-inflammatory effects of exercise," Journal of Physiology and Pharmacology, vol. 57, no. 10, pp. 43-51, 2006.

[77] K. Heikkilä, S. Ebrahim, and D. A. Lawlor, "Systematic review of the association between circulating interleukin-6 (IL-6) and cancer," European Journal of Cancer, vol. 44, no. 7, pp. 937945, 2008.

[78] T. Kishimoto, "IL-6: from its discovery to clinical applications," International Immunology, vol. 22, no. 5, pp. 347-352, 2010.

[79] T. Yokoyama, H. Osada, H. Murakami et al., "YAP1 is involved in mesothelioma development and negatively regulated by Merlin through phosphorylation," Carcinogenesis, vol. 29, no. 11, pp. 2139-2146, 2008.

[80] H. Murakami, T. Mizuno, T. Taniguchi et al., "LATS2 is a tumor suppressor gene of malignant mesothelioma," Cancer Research, vol. 71, no. 3, pp. 873-883, 2011.

[81] Y. Sekido, "Inactivation of Merlin in malignant mesothelioma cells and the Hippo signaling cascade dysregulation," Pathology International, vol. 61, no. 6, pp. 331-344, 2011. 

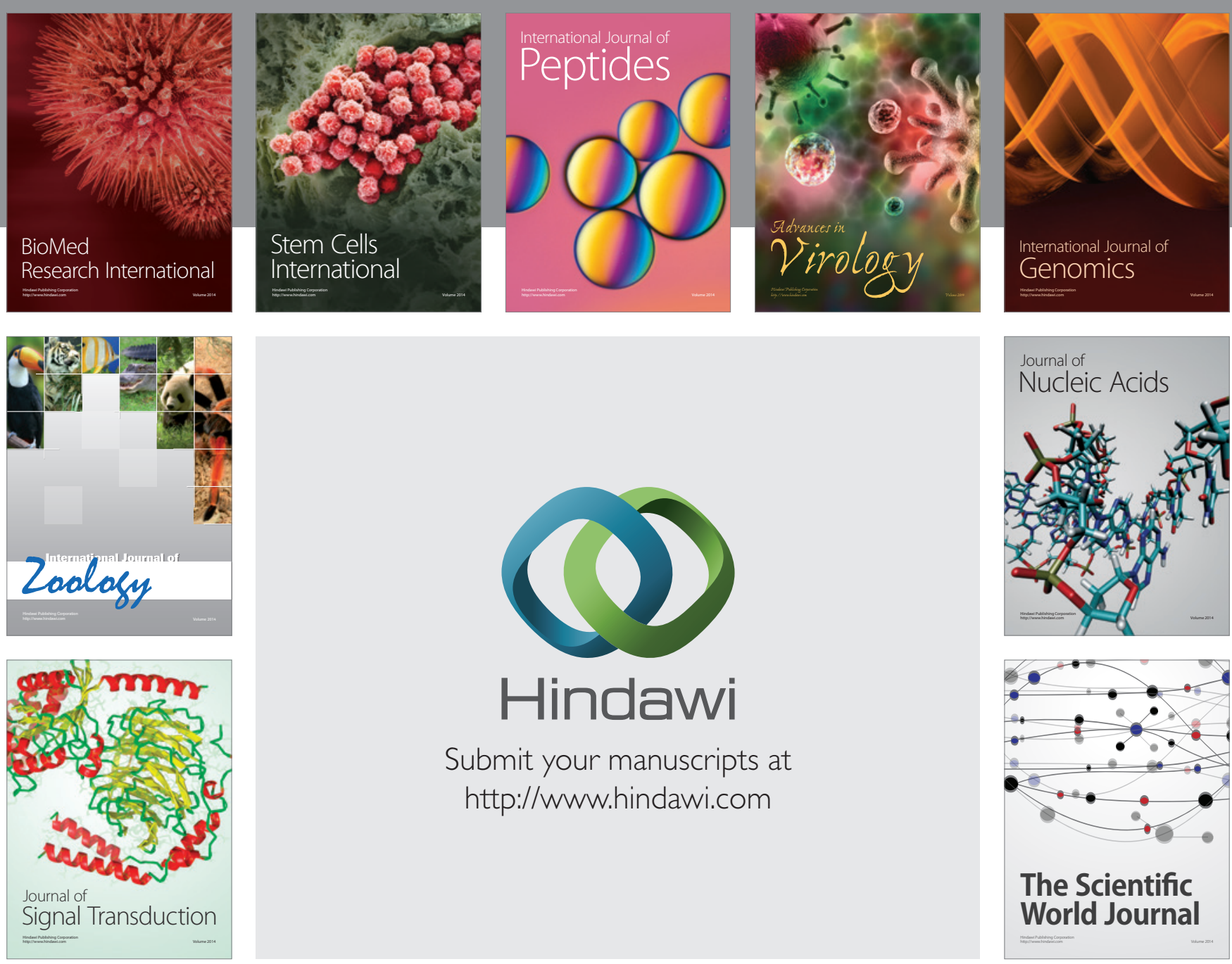

Submit your manuscripts at

http://www.hindawi.com
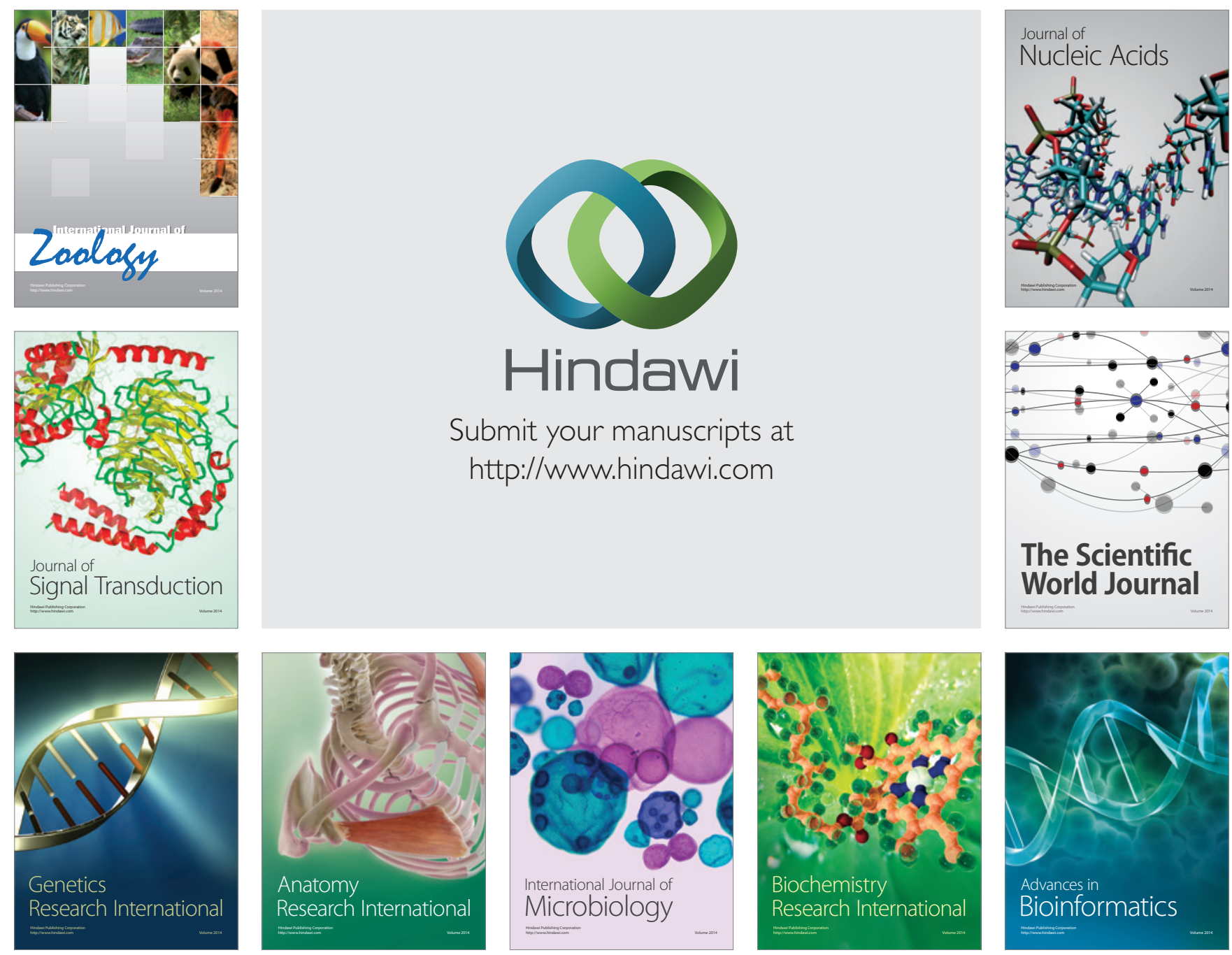

The Scientific World Journal
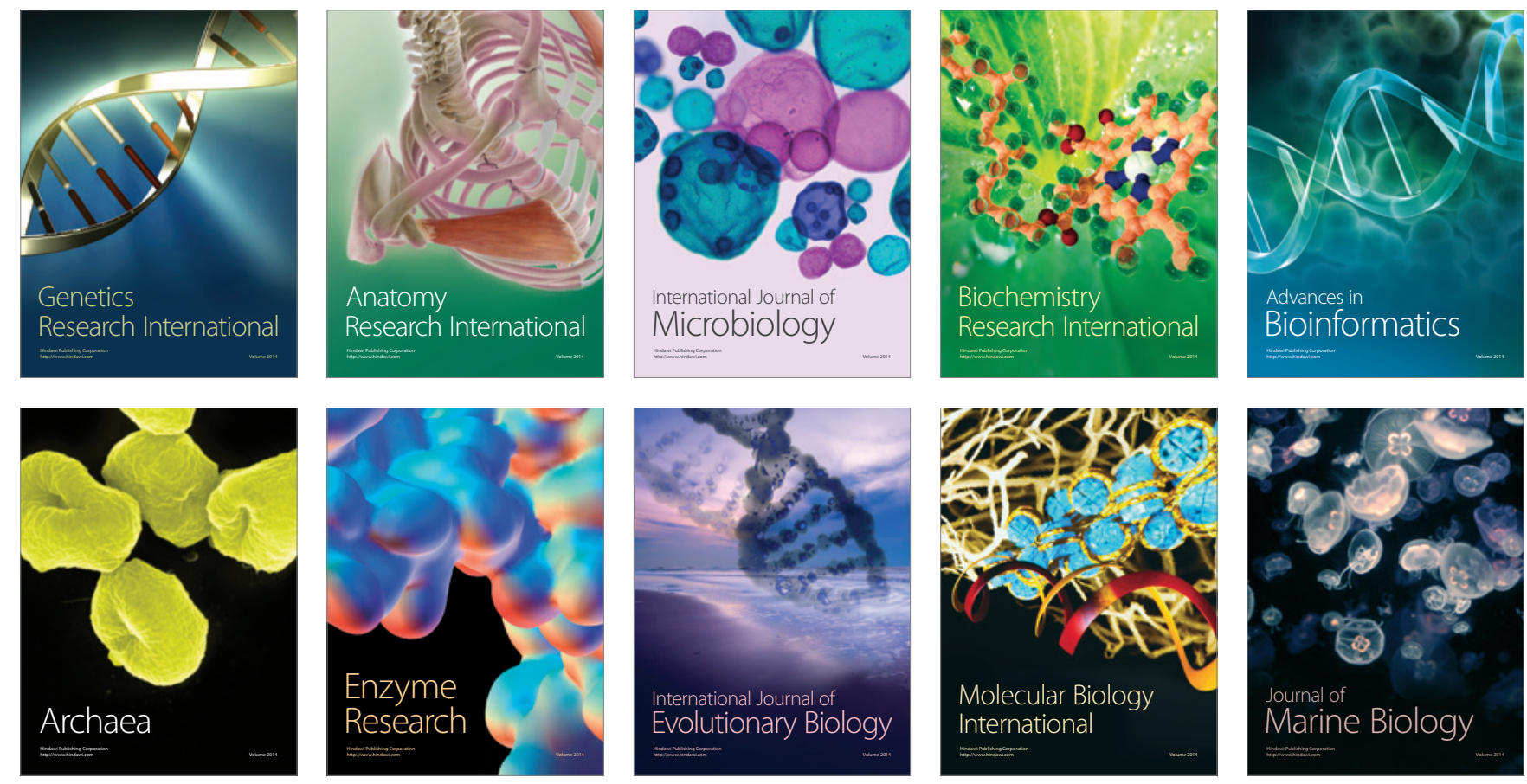